\title{
Sonological Evaluation of Malignant Nodules of Thyroid - A Study of 173 Cases
}

\author{
Sheeja G Manoj ${ }^{1}$, Visakh Prasad ${ }^{2}$ \\ ${ }^{1}$ Assistant Professor, Department of Radiodiagnosis, Sree Gokulam Medical College \& Research Foundation, Venjaramoodu, \\ TVM, ${ }^{2}$ Associate Professor, Department of Radiodiagnosis, Sree Gokulam Medical College \& Research Foundation, \\ Venjaramoodu, TVM.
}

Corresponding author: Dr Visakh Prasad, Associate Professor, Department of Radiodiagnosis, Sree Gokulam Medical College \& Research Foundation, Venjaramoodu, TVM, India

DOI: http://dx.doi.org/10.21276/ijcmsr.2019.4.4.32

How to cite this article: Sheeja G Manoj, Visakh Prasad. Sonological Evaluation of Malignant Nodules of Thyroid - A Study of 173 Cases. International Journal of Contemporary Medicine Surgery and Radiology. 2019;4(4):D134D138.

\section{A B S T R A C T}

Introduction: Thyroid ultrasound is the major diagnostic modality for evaluating thyroid nodules. The ultrasound features such as marked micro calcifications, hypoechogenicity and irregular margins were found as potential predictor for the presence of thyroid malignancies. Thus to assess the utility of USG in evaluating thyroid nodules is important. Current research objectives were to study the usefulness of ultrasound in differentiating benign and malignant thyroid nodules, to describe the thyroid nodules using ultrasound classification system and to compare the diagnostic indices for individual ultrasound features of different thyroid nodules.

Material and methods: A prospective study was conducted among 173 patients attending the Radiology department of Sree Gokulam Medical College hospital from November 2011 to June 2013. Each thyroid nodule was assessed using GE voluson 7-30 Equipment scanner with a 5-13 GHz linear probe ultrasound. The diagnostic indices of ultrasonography with FNAC/biopsy as gold standard were studied. Data was analysed using statistical software SPSS.

Results: On thyroid ultrasound examination, out of the 173 patients selected for the study, 16 of them had malignancy or suspicious for malignancy. Sensitivity of ultrasound to identify malignant thyroid nodule is $57 \%$ and specificity is $100 \%$ and the diagnostic accuracy is $93 \%$. Sensitivity of ultrasound to identify benign thyroid nodule is $95 \%$ and specificity is $96 \%$ and the diagnostic accuracy is $95 \%$.

Conclusion: Ultrasound is good diagnostic tool for evaluating the benign from malignant thyroid nodule and unnecessary biopsies/surgeries could be prevented by proper ultrasound evaluation of thyroid Nodules.

Keywords: Thyroid Nodules, Ultrasound, Diagnostic Accuracy.

\section{INTRODUCTION}

Thyroid nodules are any abnormal growth of thyroid cells that forms lump within the thyroid gland they can be sonographically discrete lesions that may be identified as normal or abnormal. Thyroid nodules are found in $4 \%$ to $8 \%$ of adults by palpation, $41 \%$ by ultrasound $50 \%$ by pathologic examination at autopsy. ${ }^{1,2}$ In contrast, compared with the very high prevalence of nodular thyroid disease, thyroid cancer is rare. Thyroid ultrasound is the major diagnostic modality for evaluating thyroid nodules. Using ultrasound, a thyroid nodule appears as a nodular lesion within the thyroid gland that is distinguishable from the adjacent parenchyma. Thyroid nodules may be single or multiple. Ultrasound features such as micro calcifications, marked hypoechogenicity and taller that wide shape have been introduced as potential prediction for the presence of thyroid malignancies. ${ }^{3}$

The prevalence of incidental thyroid carcinomas may be as high as $18 \%$ at autopsy, and $10 \%$ to $11 \%$ at surgery. 4,5 Differentiated thyroid carcinomas, including papillary (60\%to 80\%) and follicular subtypes, are most common and have a favorable prognosis. Malignant potential and behavior ranges from low-grade (papillary) to aggressive (anaplastic) and is reflected by mortality rates: papillary carcinoma $8 \%$ to $11 \%$ follicular carcinoma $24 \%$ to $33 \%$ medullary carcinoma $75 \%$ to $90 \% .{ }^{4,5}$ Prognosis is affected by gender, biologic behavior, tumor size, and the tendency for hematogenous or lymphatic metastases. ${ }^{4}$ The evaluation of a thyroid nodule depends in part on its method of discovery. If a thyroid nodule is palpable, the evaluation begins with the physical examination; if the findings are of concern, the subsequent workup may include laboratory studies, radionuclide scanning, ultrasonography, and/or fine needle aspiration (FNA). If a thyroid nodule is not palpable but detected by imaging, the workup most often either ends with the decision to observe clinically, or proceeds to an FNA, depending on the level of concern based on the imaging appearance or size. ${ }^{6}$

The challenge of managing thyroid nodules is to reassure the majority of patient who has benign disease and to 
diagnose the minority of patients who will prove to have malignant disease. ${ }^{2}$ The objectives of the study was to study the usefulness of ultrasound in differentiating benign and malignant thyroid nodules, to describe the thyroid nodules using ultrasound classification system and to compare the diagnostic indices for individual ultrasound features of different thyroid nodules.

\section{MATERIAL AND METHODS}

A prospective study was conducted among patients attending the Radiology department of Sree Gokulam Medical College hospital from November 2011 to June 2013 to study the usefulness of ultrasound in diagnosing thyroid Nodules. All patients who are willing to give consent and fulfilling the eligibility criteria were included in the study and others were excluded. The study procedure was USG examinations of each thyroid nodule were performed with - GE voluson 7-30 Equipment scanner with a 5-13 GHz linear probe. Supine position with the neck mildly hyperextended was the position for examining the patient. A small pillow can therefore be placed underneath the patient's shoulder. The neck should be examined with a high-frequency $\mathrm{MHz}$ linear array transducer. For patients with thin necks, 10 or $12 \mathrm{MHz}$ provide clear images with highest resolution, whereas in patients with more fatty necks or those with deeper lesions, lower frequencies help to achieve better tissue penetration. The thyroid gland was imaged entirely in both the transverse and longitudinal planes. Blood flow was studied using colour doppler and the relative direction of flow (toward or away from the transducer) is shown by the use of a suitable color (eg; red or blue). Level I to VI of both sides of the neck was examined for the presence of enlarged nodes Level I was evaluated in the coronal plane, levels II to VI was evaluated longitudinally (craniocaudally) and in the axial plane. All nodes that are visible on ultrasound are measured I the axial plane..$^{7,8}$

The study was commenced after obtaining the ethical clearance. The patients satisfying all the inclusion criteria were interviewed to collect the base line data relating to demographic characteristics and other relevant details. For the collection of data convenient sampling technique was used. The 173 patients for thyroid nodules were evaluated during the study period for their presence of calcification, margins, presence of circumferential halo, shape, internal composition and echogenicity. Whether the margins were irregular or smooth, (further subclassified as poorly defined or lobulated) and surrounded by a peripheral halo or not. Hypoechoic, isoechoic, hyperechoic or anechoic were the classifications of echogenecity in comparison to normal thyroid parenchyma. Based on the composition of nodules they were further classified into solid, predominantly solid $(<50 \%$ cystic change), predominantly cystic $(>50 \%$ cystic change) or cystic. If calcification was present it was categorizes as micro-calcification or macro-calcification. The ratio of antero-posterior diameter and transverse diameter were measured for determining the shape on transverse ultrasound images. ${ }^{7}$

For patient having several thyroid nodules, each of the thyroid nodules was classified separately. Multinodular goiter is a diffusely enlarged thyroid gland with multiple nodules and no intervening normal parenchyma was excluded from the study and also nodules less than $1 \mathrm{~cm}$ in size. It was because for ultrasound guided FNA the recommended minimum size is $1.5 \mathrm{~cm} .{ }^{5}$ Purely cystic type of nodules was not subjected to FNA. Then all the patients willing to enter into the study was subjected to ultrasound guided FNAC, after getting the results, it was decoded for further statistical analysis. Ultrasound scan was performed for all study participants, the results were compared with FNAC/biopsy diagnosis, as this is the gold standard.,8

For Ultrasound guided FNAC, each thyroid nodule without the administration of local anesthesia for each sample, a smear was made on 4-6 slides that was fixed in 95\% ethanol and sent to the department of pathology for papnicoloau staining. Criteria to undergo US - FNAC are:Suspicious ultrasound diagnosis, Border line ultrasound diagnosis, Insufficient cytology and Positive risk for thyroid malignancy. All FNAs were performed under ultrasound guidance and the cytopathology results were considered as the final diagnosis. For 54 of the surgically resected nodules tissue diagnosis was available. 14 of these nodules were not included in the study due to inadequate FNA and those that could not have a tissue diagnosis. The important outcome variable anticipated are diagnostic test results along with details such as number of nodules, located, size, shape, echogenicity, type of calcification etc. and the diagnosis were reported as benign or malignant.

\section{STATISTICAL ANALYSIS}

The data gathered was entered into MS.Excel sheet and SPSS V.20 software was utilized for statistical analysis. $p$ values less than $5 \%$ were taken as statistically significant value $(p<0.005)$. Diagnostic indices of ultrasonography with FNAC/biopsy as gold standard were studied.

\section{RESULTS}

Out of 173 samples selected for the study 147(84.97\%) were females and 26 (15.03\%) were male patients. According to final diagnosis, out of the 173 patients $28(16 \%)$ of them were having malignancy. After the thyroid ultrasound examination of 173 patients, each thyroid nodule was classified as follows: malignant and Suspicious for malignancy $(n=16)$, borderline $(n=18)$, and benign and probably benign $(n=139)$. Sensitivity of ultrasound to identify Malignant thyroid nodule is $57 \%$ and specificity is $100 \%$ and the diagnostic accuracy is $93 \%$. Sensitivity of ultrasound to identify Benign thyroid nodule is $95 \%$ and specificity is $96 \%$ and the diagnostic accuracy is $95 \%$. The diagnostic indices were sensitivity (57\%), specificity (100\%), positive predictive value (100\%) and negative predictive value (92\%), and accuracy (93\%) of thyroid ultrasound for differentiating a malignant lesion from a benign one. The ultrasound classification for the diagnosis of malignant and benign resulted in a high predictive value; $100 \%$ positive predictive value for malignant, $92 \%$ negative predictive value for malignant, and $99 \%$ positive predictive value for benign.

Of these patients 34 ( $\mathrm{M}: \mathrm{F}=5: 29$; mean age $=44$ years) underwent thyroid surgery due to malignant cytology results 


\begin{tabular}{|l|c|c|c|}
\hline \multirow{2}{*}{ US classifications } & Cytopathological Results & Benign \\
\cline { 2 - 4 } & Malignant & 0 & 16 \\
\hline Malignant & 16 & 7 & 18 \\
\hline Borderline & 11 & 138 & 139 \\
\hline Benign & 1 & 145 & 173 \\
\hline Total & 28 & \multicolumn{2}{c|}{} \\
\hline Fisher's exact test p-value<0.001 & \multicolumn{2}{|c}{} \\
\hline \multicolumn{2}{|r|}{ Table-1: Comparison of ultrasound diagnosis and cytopathological results } \\
\hline
\end{tabular}

\begin{tabular}{|l|c|c|c|c|}
\hline Diagnostic Indices & Sensitivity (\%) & Specificity (\%) & PPV (\%) & NPV (\%) \\
\hline Malignant & $16 / 28(57)$ & $145 / 145(100)$ & $16 / 16(100)$ & $145 / 157(92)$ \\
\hline Borderline & $7 / 145(5)$ & $17 / 28(61)$ & $7 / 18(39)$ & $17 / 155(11)$ \\
\hline Benign & $138 / 145(95)$ & $27 / 28(96)$ & $138 / 139(99)$ & $27 / 34(79)$ \\
\hline \multicolumn{4}{|r|}{ Table-2: Diagnostic indices for individual ultrasound categories } \\
\hline
\end{tabular}

\begin{tabular}{|l|l|c|c|c|c|}
\hline Ultrasound Features & Sensitivity(\%) & Specificity(\%) & PPV(\%) & NPV(\%) \\
\hline \multirow{4}{*}{ Malignant } & Hypoechoic & $16 / 28(57)$ & $145 / 145(100)$ & $16 / 16(83)$ & $145 / 157(87)$ \\
\cline { 2 - 6 } & Taller than wider & $14 / 28(50)$ & $134 / 145(92)$ & $14 / 25(56)$ & $134 / 148(91)$ \\
\cline { 2 - 6 } & Microcalcification & $15 / 28(54)$ & $143 / 145(99)$ & $15 / 17(88)$ & $143 / 156(92)$ \\
\cline { 2 - 6 } & Ill defined & $12 / 28(43)$ & $138 / 145(95)$ & $12 / 19(63)$ & $138 / 154(90)$ \\
\cline { 2 - 6 } & Centralvascularity & $16 / 28(57)$ & $134 / 145(92)$ & $16 / 27(60)$ & $134 / 146(92)$ \\
\hline \multirow{5}{*}{ Borderline } & Hypoechoic & $12 / 145(8)$ & $22 / 28(79)$ & $12 / 18(67)$ & $22 / 155(14)$ \\
\cline { 2 - 6 } & Macrocalcification & $6 / 145(4)$ & $23 / 28(82)$ & $6 / 11(55)$ & $23 / 162(14)$ \\
\cline { 2 - 6 } & Centralvascularity & $10 / 145(7)$ & $11 / 28(40)$ & $10 / 27(37)$ & $11 / 146(8)$ \\
\hline \multirow{5}{*}{ Benign } & Wider than taller & $134 / 145(92)$ & $14 / 28(50)$ & $134 / 148(91)$ & $14 / 25(56)$ \\
\cline { 2 - 6 } & a) Iso/hyper & $73 / 145(50)$ & $22 / 28(79)$ & $73 / 79(92)$ & $22 / 94(23)$ \\
\cline { 2 - 6 } & b) Hypo & $64 / 145(44)$ & $20 / 28(72)$ & $64 / 72(89)$ & $20 / 101(20)$ \\
\cline { 2 - 6 } & Well defined with Peripheral vascularity & $132 / 145(91)$ & $21 / 28(75)$ & $132 / 139(95)$ & $21 / 34(62)$ \\
\cline { 2 - 6 } & Macro/coarse calcification & $8 / 145(6)$ & $15 / 28(54)$ & $8 / 21(38)$ & $15 / 152(10)$ \\
\hline
\end{tabular}

( $n=28)$, presence of a large palpable mass with a benign cytology $(n=3)$, and patient request $(n=3)$. Repeated ultrasound -FNA was performed on thyroid nodules when the cytological results of the first ultrasound -FNA were indeterminate for malignancy $(n=18$. The nodules $(n=139)$ categorized as the benign in ultrasound and benign cytology were followed up with ultrasound examination at least after 12 months. There can be a significant interval changes, if there is a new detection of suspicious ultrasound features or significant increase in nodular size $(50 \%$ or more increase in nodular volume) was not found. Out of the 173 patients, $34 \%$ exhibited solitary thyroid nodule and $66 \%$ had two or more thyroid nodules.

All of the nodules categorized as malignant on thyroid ultrasound, were confirmed to be papillary thyroid carcinomas (PTCs), except one which was a medullary thyroid carcinoma. Nodules that were suspicious for malignancy was 16 which include 10 PTCs, 4 pseudonodules related to thyroiditis, one was a benign lesion confirmed as a spontaneous collapse of a previously benign cystic nodule, and the last one was a nodular hyperplasia.

\section{DISCUSSION}

Thyroid nodular diseases are the once which are characterized by the presence of one or more palpable or non-palpable nodules within the substance of the thyroid gland. ${ }^{9}$ High- resolution thyroid ultrasound is considered to be the most useful and efficient diagnostic tool for evaluating thyroid nodules. Several studies had reported the diagnostic accuracy of thyroid ultrasound for thyroid nodules, and several other ultrasound characteristics had been introduced as potential predictors for thyroid malignancy. In many reports, there is a significant overlap of the ultrasound characteristics between benign and malignant thyroid nodules. In addition, these studies are either retrospective or do not include a systemized diagnostic classification for thyroid ultrasound..$^{9,10}$

Kim et al. reported that the sensitivity of thyroid ultrasound is high for non-palpable thyroid nodules. The ultrasound findings of benign and malignant lesions in a nonpalpable thyroid nodule are different and thus help in their differentiation. ${ }^{11}$ Lee et al believe that there are several reasons behind the high diagnostic accuracy of thyroid ultrasound in his study. First, he used an ultrasound classification system with 5 categories. Second, he used real-time ultrasound and a relatively higher resolution ultrasound modality compared with the previous study. Lastly, the sonographic features of associated lymph nodes in favor of malignancy were the existence of lymphadenopathy with cystic components or intranodal micro calcifications. ${ }^{12}$ Recently, Horvath et al. have reported that an ultrasonography-based reporting system improved patient management and cost-effectiveness by helping avoid unnecessary FNA. ${ }^{13}$ 
In our study, after the thyroid ultrasound examination of 173 patients, each thyroid nodule was classified as follows: malignant and Suspicious for malignancy $(\mathrm{n}=16)$, borderline $(n=18)$, and benign and probably benign $(n=139)$. Sensitivity of ultrasound to identify Malignant thyroid nodule is $95 \%$ and specificity is $96 \%$ and the diagnostic accuracy is $95 \%$. Sensitivity of ultrasound to identify Benign thyroid nodule is $95 \%$ and specificity is $96 \%$ and the diagnostic accuracy is $95 \%$. The sensitivity (57\%), specificity (100\%), positive predictive value (100\%) and negative predictive value (92\%), and accuracy (93\%) of thyroid ultrasound for differentiating a malignant lesion from a benign one. The assigned ultrasound categories for the diagnosis of malignant and benign shown a high predictive value; $100 \%$ positive predictive value for malignant, 92\% negative predictive value for malignant, and 99\% positive predictive value for benign.

There were an overall higher percentage of malignant nodules in our study $(16.18 \%)$ than has been previously reported in other studies. ${ }^{9,14}$ However, this can be due to the possibility of a bias in our study toward inclusion of nodules with high suspicion of malignancy. Women encounter more thyroid problems than men leads to an increased incidence found in women than in men in our study. Studies have reported that most malignancies are found in a hypoechoic nodule, but due to the high prevalence of benign lesions most of the hypoechoic nodules are benign lesions. ${ }^{15}$ In our study also, all the malignant nodules (100\%) were hypoechoic in appearance, though the majority of the hypoechoic nodules were benign (46\%). We found a low sensitivity (57\%) but an appreciable specificity (100\%) of marked hypoechogenecity for malignant thyroid nodules. Marked hypoechogenecity in ultrasound has a high negative predictive value (91.9\%).

In this study showed that, irregular margins or poorly defined margins had a high negative predictive value $(96.1 \%)$ and a high diagnostic accuracy (87.9\%) among the malignant nodules in ultrasound. In various other studies reported that the sensitivity of ill-defined margins or irregular margins in the diagnosis of malignant nodules ranges widely from $8.3 \%$ to $77.5 \% .{ }^{5,16}$ In our study, the occurrence of calcification more common in malignant nodules (94\%) than in benign nodules (6\%). The nodules for presence of microcalcification as well as macrocalcification were assessed. Kim et al. ${ }^{17}$ classified the macrocalcifications as solitary calcifications, eggshell calcification and the not-otherwise-specified (NOS) variety. The ultrasound findings of microcalcifications were highly specific of malignant thyroid nodules along with a high positive predictive value.

\section{CONCLUSION}

This study was conducted to study the usefulness of ultrasound in diagnosing thyroid Nodules. Out of 173 samples selected for the study $84.97 \%$ were females and $15.03 \%$ were male patients. 28 samples (16.2\%) had confirmed malignancy.After the thyroid US examination of 173 patients, each thyroid nodule was classified as follows: malignant and Suspicious for malignancy $(\mathrm{n}=16)$, borderline $(\mathrm{n}=18)$, and benign and probably benign $(n=139)$. Sensitivity of ultrasound to identify malignant thyroid nodule is $57 \%$ and specificity is $100 \%$ and the diagnostic accuracy is $93 \%$. Sensitivity of ultrasound to identify benign thyroid nodule is $95 \%$ and specificity is $96 \%$ and the diagnostic accuracy is $95 \%$. Sensitivity of US to identify hypo echoic features in malignant thyroid nodule is $57 \%$, specificity is $100 \%$ and diagnostic accuracy is $93 \%$. Hence ultrasound is good diagnostic tool for evaluating the benign from malignant thyroid nodule and unnecessary biopsies/surgeries could be prevented by proper ultrasound evaluation of thyroid Nodules.

\section{REFERENCES}

1. Horlocker T, Hay I. Prevalence of incidental nodular thyroid disease detected during high-resolution parathyroid sonography. In: Medeiros Neto G, Gaitan E, eds. Frontiers in Thyroidology. New York: Plenum Medical; 1985;2(3): 1309-12.

2. Mortenson J, Woolner L, Bennett W. Gross and microscopic findings in clinically normal thyroid glands. J Clin Endocrinol Metab. 1955; 15(2);1270-80.

3. Kim EK, Park CS, Chug WY, oh kk. Kim DI, Lee JT, et al. New sonographic criteria for recommending fineneedle aspiration biopsy of nonpalpable solid nodules of the thyroid. AJR Am J Roentgenol 2002;178(1);687-91

4. Jemal A, Murray T, Ward E, et al. Cancer statistics, 2005. CA Cancer J Clin. 2005; 55(4);10-30.

5. Gilliland F, Hunt W, Morris D, et al. Prognostic factors for thyroid carcinoma: a population-based study of 15, 698 cases from the surveillance, epidemiology and end results (SEER) Program 1973-1991. Cancer. 1997; 79(5);564-73.

6. Tae HJ, Lim DJ, Baek KH, Park WC, Lee YS, Choi $\mathrm{JE}$, et al. Diagnostic value of ultrasonography to distinguish between benign and malignant lesions in the management of thyroid nodules. Thyroid 2007;17(3):461-66

7. Morris LF, Ragavendra N, Yeh MW. Evidence-based assessment of the role of ultrasonography in the the management of benign thyroid nodules. World J Surg 2008; 32(7):1253-63.

8. Mandel SJ.Diagnostic use ultrasonography in patients with nodular thyroid disease. Endocr Pract 2004;10 (3):246-52.

9. Solbiati L, Charboneau JW, Osti V, James EM, Hay ID. The Thyroid Gland. In: Wilson SR, Charboneau JW, Rumack CM, editors. Diagnostic Ultrasound. $3^{\text {rd }}$ ed. Missouri: Mosby, Elsevier Inc.; 2005; pg: 735-70.

10. Mortenson J, Woolner L, Benneu W. Gross and microscopic findings in clinically normal thyroid glands. J Clin Endocrinol Metab 1955;15(5):1270-80.

11. Kim EK, Parek CS, Chung WY, et al. New sonographic criteria for recommending fine-needle aspiration biopsy of non-palpable solid nodules of the thyroid. AJR Am J Roentgenol. 2002;178(5):687-91.

12. Lee YH, Kim DW, In HS, Park JS, Kim SH, Eom JW, Kim B, Lee EJ, Rho MH. Differentiation between benign and malignant solid thyroid nodules using an US classification system. Korean J Radiol. 2011;12(5):55967.

13. Horvath E, Majlis S, Rossi R, Franco C, Niedmann JP, Castro A, et al. An ultrasonogram reporting system for thyroid nodules stratifying cancer risk for clinical management. J Clin EEndocrinol Metab 2009;94(6); 
pg:1748-51.

14. Frates MC, Benson CB, Charboneau JW, Cibas ES, Clark OH, Coleman BG, et al. Management of Thyroid Nodules Detected at US: society of Radiologists in Ultrasound Consensus Conference Statement. Radiology 2005;237(1):794-800.

15. Bonavita JA, Mayo J, Babb J, Bennett G, Oweity T, Macari M, et al. Pattern recognition of benign nodules at ultrasound of the thyroid: Which nodules can be left alone? AJR Am J Roentgenol 2009;193(3):207-13.

16. Cope $\mathrm{O}$, Crelin E, Rawson R,et al. The thyroid glandthe parathyroid glands. In:Netter F, editor. The CIBA Collection of Medical Illustration, Vol.4. Endocrine System and Selected Metabolic Diseases. New York: CIBA pharmaceutical Company; 1965.p.41-73.

17. Kim MJ, Kim EK, Kwak JY, Park CS, Chung WY, Nam KH. Differentiation of thyroid nodules with macrocalcifications role of suspicious sonographic findings. J Ultrasound Med 2008;27(6):1179-84.

Source of Support: Nil; Conflict of Interest: None

Submitted: 07-11-2019; Accepted: 03-12-2019; Published online: 18-12-2019 